\title{
Spin Wave Dynamics of 2D and 3D Heisenberg Antiferromagnets
}

\author{
R.A. Cowley, D.A. Tennant* and R. Coldea \\ Clarendon Laboratory, University of Oxford \\ Parks Road, Oxford OX1 3PU, UK
}

\begin{abstract}
The excitations of the 2D Heisenberg antiferromagnet, $\mathrm{Rb}_{2} \mathrm{MnF}_{4}$, were studied using neutron scattering techniques with the MAPS spectrometer at the ISIS facility of Rutherford Appleton Laboratory. Measurements were made of the magnetic excitations over the whole 2D Brillouin zone at 6 temperatures below the ordering temperature of $38 \mathrm{~K}$ and 6 temperatures above. It was found that the excitations were well defined if their wave vectors were larger than the inverse correlation length and were overdamped if the wave vectors of the excitations were smaller than the inverse correlation length. In more detail we have compared our experimental results with the results of classical simulations and the results gave a very adequate description of the experimental results except at the lowest temperature where the form of the dispersion relation was correct but the energies of the excitations were in error. Nevertheless, classical simulations do provide an efficient and easily implemented methodology for modelling the excitations in the Heisenberg magnets. The damping of the excitations was experimentally found to follow a $T^{2}$ behaviour over all wave vector and energy scales. This is in agreement with the classical simulations but inconsistent with analytic theories of the damping for the 2D Heisenberg model and in particular does not agree with hydrodynamic behaviour or dynamic scaling. The result is similar to that found in 3D Heisenberg systems and suggests that more analytic theory is needed to explain the experimental results for both 2D and 3D Heisenberg magnets.
\end{abstract}

PACS numbers: 75.40.Gb, 75.50.Ee, 61.12.Ex

\section{Introduction}

The spin wave dynamics of both two- and three-dimensional antiferromagnets with spin $S=5 / 2$ are discussed in this article by comparing the results of neutron scattering measurements on $\mathrm{Rb}_{2} \mathrm{MnF}_{4}$ and $\mathrm{RbMnF}_{3}$ with computer simulations and analytical theories. Surprisingly in both materials the classical computer simulations give a good account of the measurements whereas the analytical calculations fail to give a satisfactory description and we suggest they require further development. The results for the $3 \mathrm{D}$ system $\mathrm{RbMnF}_{3}$ were published about 10 years ago $[1,2]$ whereas the results for the $2 \mathrm{D}$ system, which are more dramatic, have been recently published [3]. We shall therefore in this article concentrate on describing the results for the 2D system and only briefly review the $3 \mathrm{D}$ results.

\section{Experimental measurements}

The measurements of the spin excitations for the single crystal of $\mathrm{Rb}_{2} \mathrm{MnF}_{4}$ were made using neutron scattering techniques with the MAPS spectrometer at the ISIS facility of the Rutherford Appleton Laboratory whereas the measurements on the single crystal of $\mathrm{RbMnF}_{3}$ were

\footnotetext{
* Current address: Hahn Meitner Institute, Glienicker Str. 100,
} D-14109 Berlin, Germany. made using triple crystal spectrometers at Ris $\varnothing$ and at the Institut Laue Langevin. One of the objectives was to find out how successful the MAPS spectrometer would be to perform this type of measurement.

The structure of $\mathrm{Rb}_{2} \mathrm{MnF}_{4}$ is shown in Fig. 1a and is the same as that of the superconductor $\mathrm{La}_{2} \mathrm{CuO}_{4}$ with $\mathrm{Mn}-\mathrm{F}_{2}$ layers separated from one another by $\mathrm{Rb}-\mathrm{F}$ layers along the $c$-axis. The spin waves of $\mathrm{Rb}_{2} \mathrm{MnF}_{4}$ have been measured $[4,5]$ and the exchange is dominated by nearest neighbour interactions within the magnetic planes. There is, however, a weak magnetic coupling between the layers due to dipolar effects which results in long range antiferromagnetic ordering below $38.4 \mathrm{~K}$. If there was no coupling between the planes, the Mermin-Wagner theorem would prohibit long range order at any non-zero temperature. The Curie-Weiss temperature is $87.5 \mathrm{~K}$ which is very considerably above the ordering temperature so there is a large range of temperature where fluctuations dominate the ordering.

The MAPS spectrometer is a direct geometry time-of-flight instrument equipped with $16 \mathrm{~m}^{2}$ of position sensitive detectors which can be divided into 40,000 different spatial channels. The sample was placed into a variable temperature cryostat and data collected for 12 temperatures between $9 \mathrm{~K}$ and $100 \mathrm{~K}$. The sample was oriented so that the $c$-axis was in the horizontal scattering plane and data was collected with an incident neutron energy of $24.94 \mathrm{meV}$ and a chopper speed of $300 \mathrm{~Hz}$. Since there is 

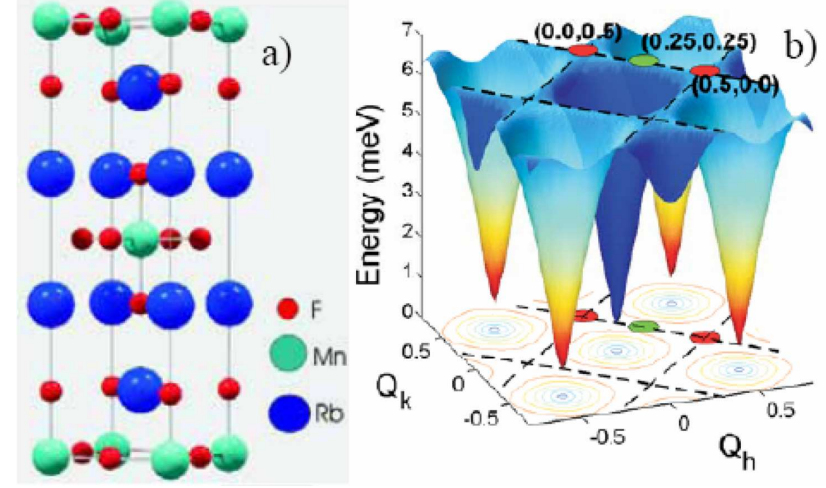

Fig. 1. Part (a) shows the structure of $\mathrm{Rb}_{2} \mathrm{MnF}_{4}$ while part (b) shows the dispersion relation at low temperature as a function of $Q_{h}, Q_{k}$. The red colouring shows the maximum intensity and the blue colouring lower intensity.

little correlation between the planes the $L$ component of the wave vector can be projected out enabling the measurements to give the scattering in $(H, K, \omega)$ space as illustrated at low temperatures in Fig. 1b. The advantage of the MAPS spectrometer is that all the data is obtained over the whole Brillouin zone at the same time so that data collection at a range of different temperatures is efficient and not too time consuming. The disadvantage is that the experimental resolution improves with the energy transfer so that high energy spin waves are measured with better resolution than the low energy ones. Ideally it would be preferable if low energy spin waves were measured most accurately and this is readily achieved with a triple axis spectrometer but the efficiency of the data collection is then reduced. The data collected with MAPS was analysed by extracting the results as at constant wave vector and then fitting a Lorentzian profile to the scattering convoluted with the experimental resolution. The resulting energies and widths of the excitations were then compared with the simulations and the theoretical models.

\section{Classical simulations}

Numerical simulations were performed using a classical model with a procedure similar to that of Metropolis $[3,6]$. The energy is calculated classically for each spin configuration which means that the spins are assumed to have unit length and to be directed in any direction. The configuration is up-dated by allowing one or more spins to change their orientation and then considering whether the energy has increased or decreased. Unfortunately, this procedure is slow and so the calculations used an over-relaxation algorithm which uses configurations that are as far as possible from the previous configuration. This enables the procedure to be speeded up by many orders of magnitude.

One of the main difficulties is how to choose the parameters, $J_{\mathrm{c}}$ and $S_{\mathrm{c}}$ for the simulations so as to have a classi- cal description of a quantum system. We have chosen to fix the parameters at either high or low temperature. The integral over all energies and the whole Brillouin zone is proportional to $S(S+1)$ for a quantum system and to $S_{\mathrm{c}}^{2}$ for a classical system at all temperatures. Furthermore, the integral of the scattering for each individual wave vector at high temperatures gives the same relation be-

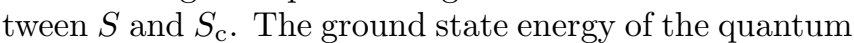
system and the classical system neglecting the zero point energy is the same if $S_{\mathrm{c}}^{2}=S(S+1)$. A difficulty however arises when we consider the expression for the spin wave energy at zero temperature. The quantum system gives an energy proportional to $S$ with a possible correction from the non-linear interactions of $(1+0.157 /(2 S))$, whereas for a classical system the energy of the excitations is proportional at low temperatures to $S_{\mathrm{c}}$. If therefore we use $S_{\mathrm{c}}^{2}=S(S+1)$ there will be a deviation at low temperatures for the spin wave energies. We have therefore done two simulations, model $A$ has $S_{\mathrm{c}}^{2}=S(S+1)$ with $J_{\mathrm{c}}=J=0.63 \mathrm{meV}$ which should work well at high temperature but deviate from the quantum result at low temperature and model $B$ which has $S_{\mathrm{c}}=S$ and $J_{\mathrm{c}}=0.65 \mathrm{meV}$ which agrees with the low temperature energies but is expected to deviate from the high temperature results. Our results will show that model $A$ gives a much better description overall except at low temperatures.

\section{Results}

It is convenient to begin our comparison of the experimental results with the simulations at high temperature. The neutron scattering measurements shown in Fig. 2 were obtained at $100.7 \mathrm{~K}$ when the scattering can be described by quasi-elastic scattering. In Fig. 2 the results are compared with both model $A$ and model $B$ of the simulations and it is clear that model $A$ gives substantially better agreement with the experimental results. This is not surprising because model $A$ had the parameters adjusted to agree exactly with the integrated intensity at high temperature. The results show considerable scatter at the elastic position. This is because of the relatively large incoherent scattering that was subtracted from the elastic position. Figure 3 shows similar experimental results at $62.8 \mathrm{~K}$ when the scattering has a more complicated profile. The results of the simulations for models $A$ and $B$ are both shown and it is again clear that model $A$ gives the best agreement with the experimental results. The results shown in Figs. 2 and 3 are restricted to only a few wave vectors at the zone boundary. In order to show the results for many different wave vectors we must compress the data. This is done for the results at $62.8 \mathrm{~K}$ throughout the Brillouin zone in Fig. 4, by showing the energy and widths of the excitations and comparing the results with a similar analysis for mo$\operatorname{del} A$. Both the peak positions and the widths for the simulations are slightly larger than for the experiments but generally the results are in good agreement for all 


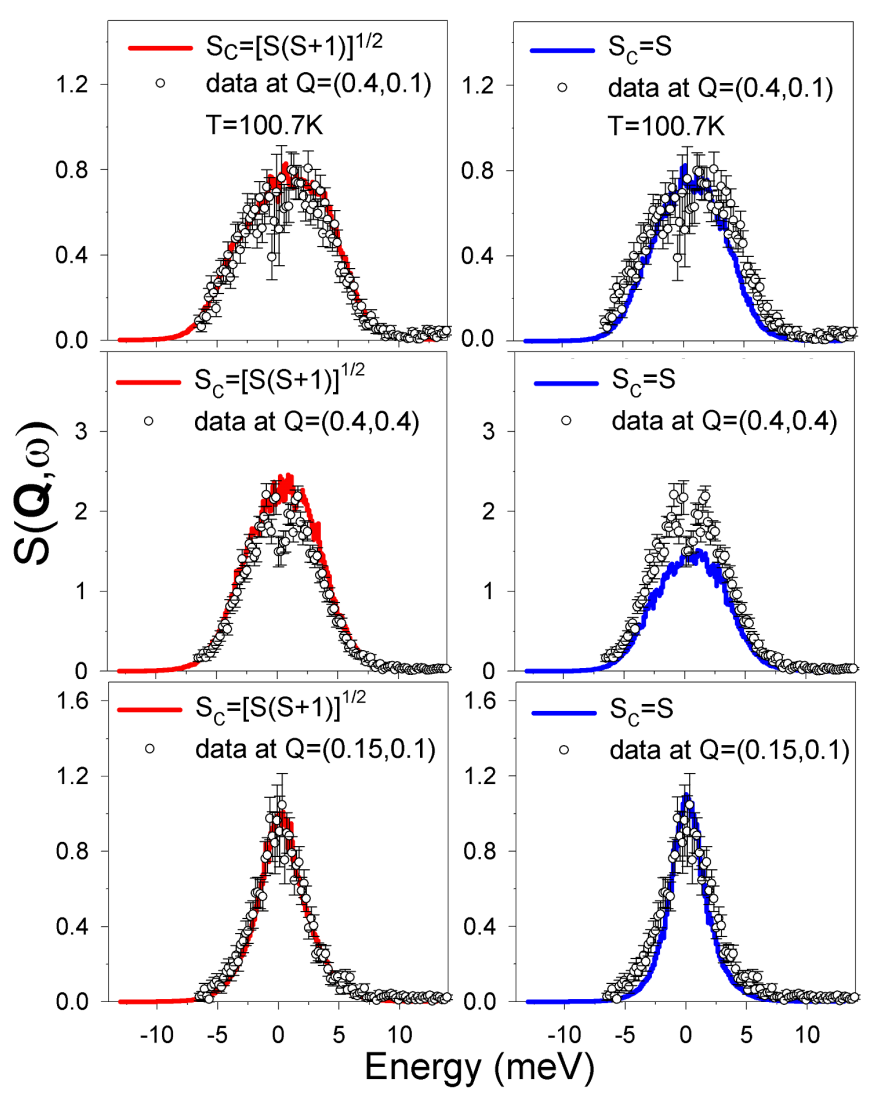

Fig. 2. The experiment data and predictions of models $A$ and $B$ simulations at $100.7 \mathrm{~K}$.

wave vectors. Similar results are shown in Fig. 5 for the results of the experiments and simulations for $46.9 \mathrm{~K}$.

Below the phase transition at $30.2 \mathrm{~K}$ the widths of the excitations are smaller and the energies are becoming larger as shown in Fig. 6. Model $A$ still gives a better description of the data than model $B$ but in parts of the Brillouin zone the errors on the damping are becoming significant. At lower temperatures the picture does not change appreciably although the experimental width is unreliable and the energy of the excitations is becoming larger than the experimental energy as discussed above. Our conclusion is that the classical simulations with model $A$ provide an excellent description of the experiments except at the lowest temperatures where the omission of the zero point fluctuations in the classical simulations results in them having a larger energy than the experiment.

The results for the zone boundary excitations are summarised in Fig. 7 for a range of temperatures and compared with the simulations and the agreement is very good. Figure 7 also compares our measurements with the results from analytical calculations. The energy of the excitations is well described up to a temperature of $30 \mathrm{~K}$ by the random phase approximation. The damping is, however, more complex and the theory of Tyc and Halperin [7] is reasonably satisfactory for the zone bound-

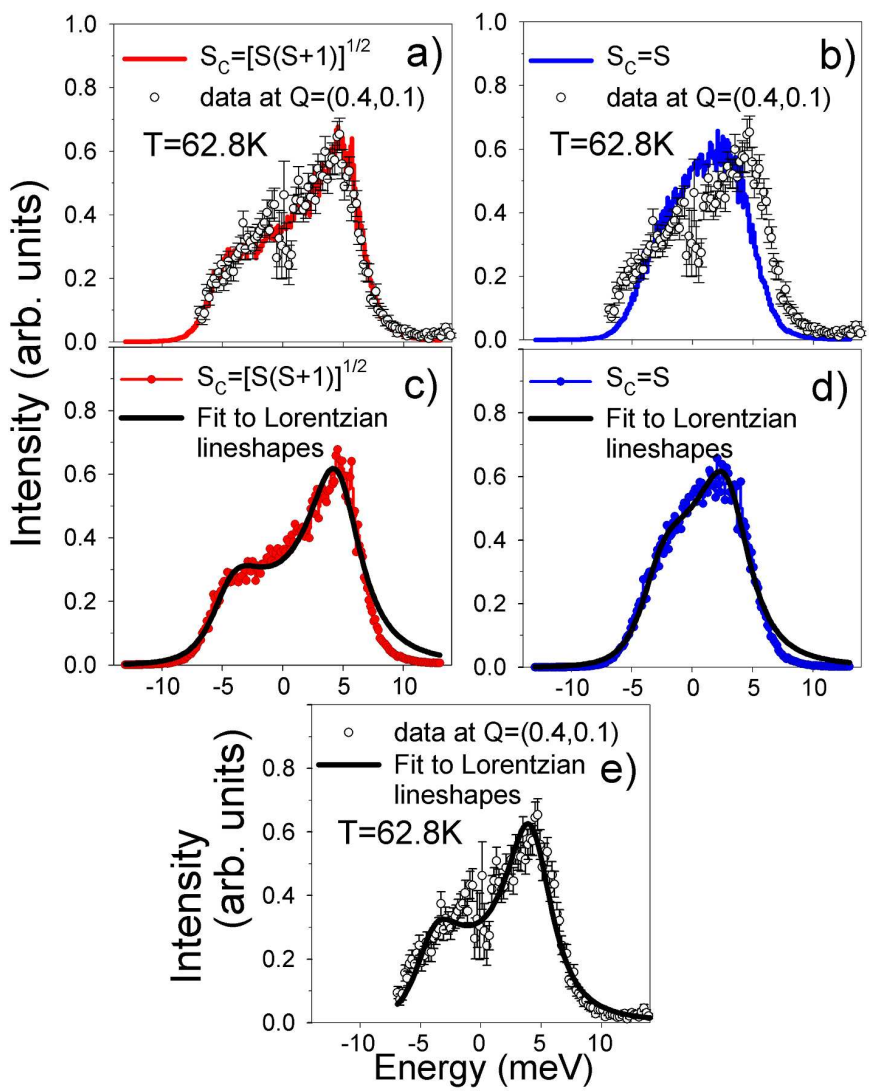

Fig. 3. The experimental data and the predictions for models $A$ and $B$ of the simulations at a temperature of $62.8 \mathrm{~K}$.

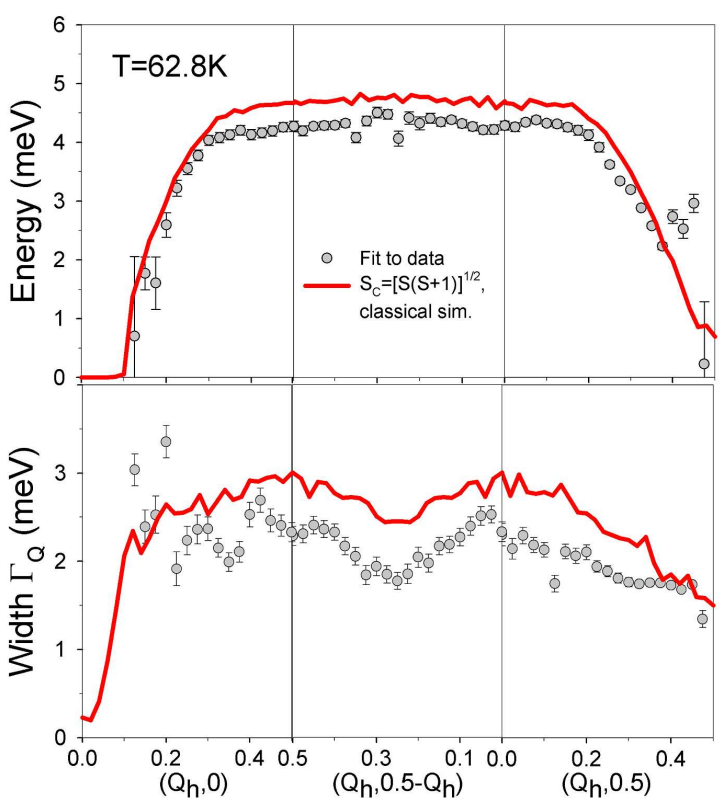

Fig. 4. The energy and width of the excitations at $62.8 \mathrm{~K}$ (black points) compared with the results for model $A$ (red points). 


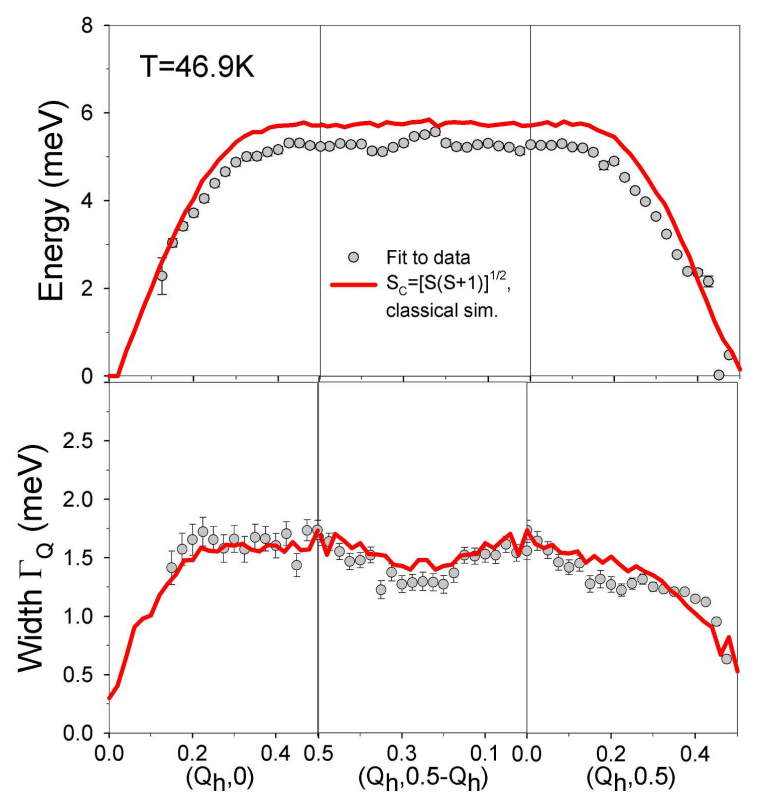

Fig. 5. The energy and width of the excitations at $46.9 \mathrm{~K}$ compared with the results of model $A$ (red points).

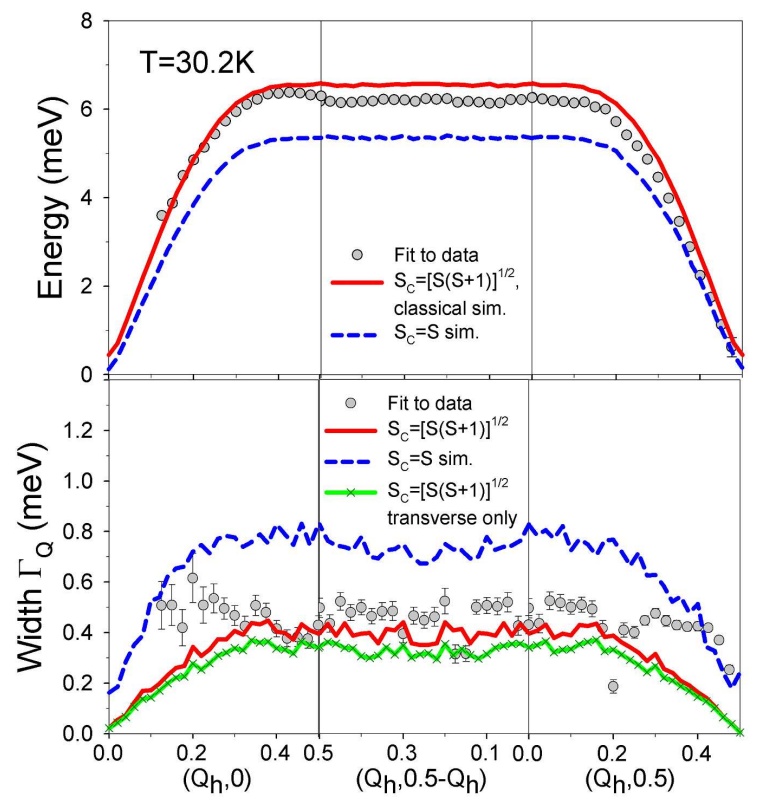

Fig. 6. The energies and widths of the excitations at $30.2 \mathrm{~K}$ compared with the simulations. The red points show the predictions of model $A$, the blue the predictions of model $B$ and green is the transverse component only of model $A$.

ary. In more detail the zone boundary damping is given by the theory of Kopietz [8]. His theory suggests that the damping is proportional to $T^{3}$ multiplied by factors that are independent of temperature but vary with wave vector. We have examined this prediction for our data and find that our experimental results shown in Fig. 7

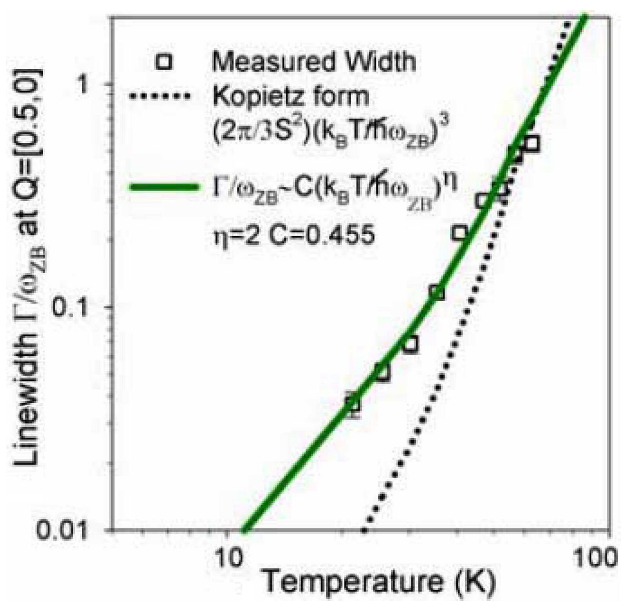

Fig. 7. A logarithmic plot showing that the temperature dependence of the zone boundary width divided by the energy is proportional to $T^{2}$ whereas the theory of Kopietz gives the width as proportional to $T^{3}$.

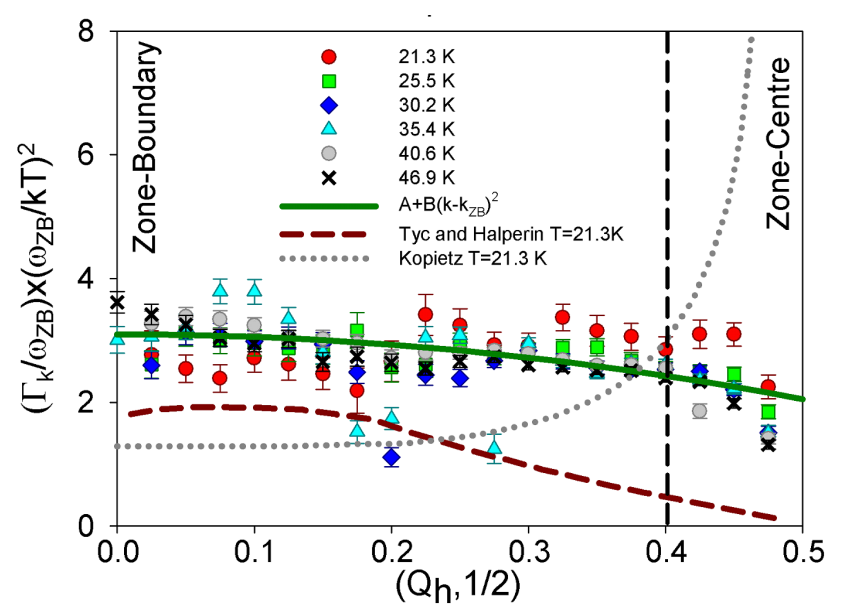

Fig. 8. The damping divided by the energy and $T^{2}$ showing that there is only a slight downward trend at the zone centre. The predictions of this for the theories of Tyc et al. are shown at a temperature of $21.3 \mathrm{~K}$ and neither is satisfactory.

and the simulations behave as $T^{2}$. In Fig. 8 we show the line width divided by the energy of the excitation and $T^{2}$ as a function of the wave vector. The data within error all lie on a common curve which has only a slight downward trend. In contrast the theories for $21.3 \mathrm{~K}$ have considerable dispersion not observed in the experimental results.

\section{5. $\mathbf{R b M n F}_{3}$}

$\mathrm{RbMnF}_{3}$ is a cubic perovskite and so an ideal example of a system with three components $n=3$ and $D=3$. The critical scattering close to the phase transition was measured in the early 1970's and we repeated and improved the measurements in the late 1990's using triple axis crystal spectrometers; TAS7 at Ris $\varnothing$ and IN14 at 


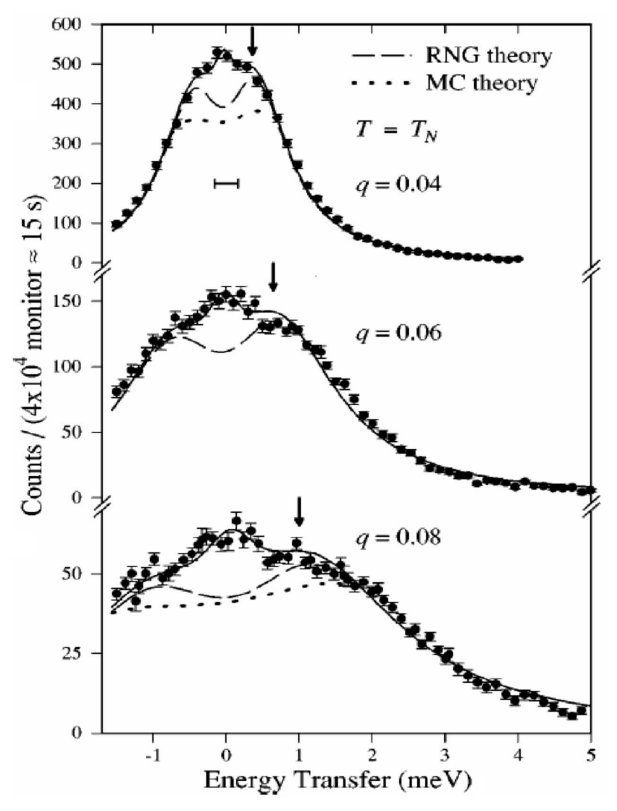

Fig. 9. Scattering at $T_{\mathrm{N}}$ for $\mathrm{RbMnF}_{3}$. The experimental profile shows a maximum at zero energy whereas the renormalisation group theory and the mode coupling theory show minima.

the ILL. Measurements were made of the scattering near the $(1 / 2,1 / 2,1 / 2)$ antiferromagnetic ordering wave vector at temperatures between $0.77 T_{\mathrm{N}}$ and $1.11 T_{\mathrm{N}}$ where $T_{\mathrm{N}}$ was measured as $82.8 \mathrm{~K}$. The purpose of our measurements was to study the dynamics of $\mathrm{RbMnF}_{3}$ in detail and in particular to determine if it could be understood by renormalisation group theory. The main result of the paper is shown in Fig. 9 which shows the scattering at $T_{\mathrm{N}}$ for 3 different wave vectors. For $q=0.04$ the line shape is consistent with being a single peak but at larger wave vectors the scattering clearly consists of 3 distinct peaks. Also shown in the figure are the predictions of the line shape calculated from renormalisation group theory [9] and from mode coupling theories [10]. For these models we have fitted the data to the measurements at large energy transfers when both these theories give insufficient intensity at low energy transfers. We therefore fitted the results to an inelastic component and a quasi-elastic component and found that the energy width of both these components scaled as $q^{z}$ with $z=1.43 \pm 0.04$ which is consistent with the theoretical value of the exponent $z=1.5$.

Most of the other properties of $\mathrm{RbMnF}_{3}$ behaved as predicted by renormalisation group theory except for the behaviour of the longitudinal susceptibility below $T_{\mathrm{N}}$. The energy width of the longitudinal width is predicted to behave as $q^{2}$. The experiments were very difficult because the longitudinal scattering could only be measured above $0.936 T_{\mathrm{N}}$ and not close to $q=0$ than 0.02 because of the intense transverse scattering. Nevertheless, the energy width was found to behave as $q^{1.58 \pm 0.03}$ which is clearly different from predictions.

Numerical simulations of these results were performed by Tsai et al. [2]. The results shown in Fig. 10 are their calculations of the scattering at $T_{\mathrm{N}}$ and clearly there is a quasi-elastic component as well as the inelastic component. We consider that if experiments and simulations agree with one another at least semi-quantitatively, then the results are correct for real systems even though renormalisation group theory and mode coupling theory disagree with the results.
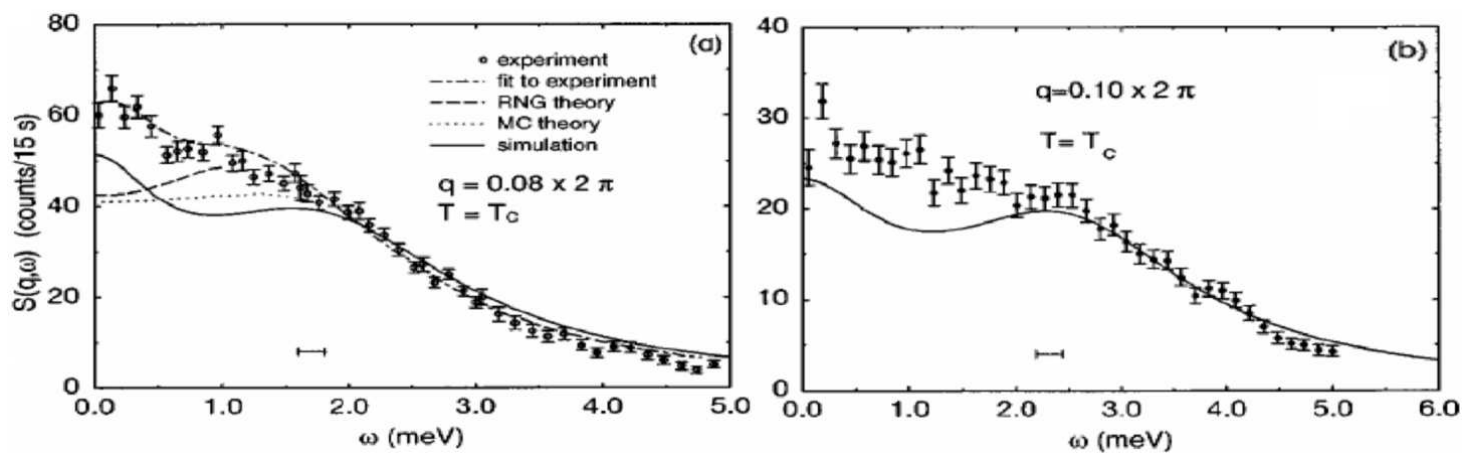

Fig. 10. The computer simulations of Tsai et al. [2] compared with the experimental results. Both give maxima at zero energy transfer.

\section{Conclusions}

The results for both $\mathrm{RbMnF}_{3}$ and $\mathrm{Rb}_{2} \mathrm{MnF}_{4}$ suggest that the analytical results for the spin waves do not agree with the experimental results from neutron scattering and not with the results of simulations using a classical model. We shall first discuss $\mathrm{RbMnF}_{3}$. For this material there have been several measurements over the last 50 years of the exponents and the universal amplitude ratios. These largely gave results that within error agree with the theoretical predictions. The discrepan- 
cies occurred with the shape of the scattering at $T_{\mathrm{N}}$ for which the experiments and computer simulations gave a three peak structure while the shape calculated by renormalisation group theory has only two peaks. Secondly there is possibly a discrepancy below $T_{\mathrm{N}}$ for the quasi-elastic longitudinal scattering. We consider that both of these may well be connected. The scattering profile is continuous as the temperature is varied and our results show a longitudinal peak below $T_{\mathrm{N}}$ which evolves to the quasi-elastic peak in the scattering above $T_{\mathrm{N}}$. This is the peak that is missing from the analytic theories. Consequently, we suggest that the theory should be reworked so as to include the longitudinal-like contributions above $T_{\mathrm{N}}$. Possibly this contribution is large in both the experiments and the simulations because $\mathrm{RbMnF}_{3}$ is cubic and the analytic theories are evaluated for an isotropic system.

In the case of $\mathrm{Rb}_{2} \mathrm{MnF}_{4}$ the discrepancies between the analytic theories and the experiments and simulations occur over a much wider temperature and wave vector range. Nevertheless, static scattering measurements of the correlation length [11] agree with the theoretical work of Hasenfratz [12]. The dynamics at small wave vectors and low energies were found to be consistent with scaling but the temperature dependence of the scaled frequency was found to be different from that expected [13]. These measurements were made with an applied field so as to reduce the anisotropy. The measurements we have made are over a much wider range of temperatures and of wave vectors. One of our main conclusions is that classical simulations, model $A$, provide an excellent description of the measurements except for the very low temperature spin waves. We suggest that the agreement with analytic theories is less satisfactory. The damping of the excitations scales as $T^{2}$ instead of the predicted $T^{3}$ and the damping divided by the energy of the excitations is approximately constant throughout the Brillouin zone. Further devel- opment of the analytic theories is needed to explain these results.

\section{Acknowledgments}

We are grateful to all our collaborators in this work: T.G. Perring, D.F. McMorrow, B. Roessli, T. Huberman and C.D. Frost and for financial support from the EPSRC and the CCLRC.

\section{References}

[1] R. Coldea, R.A. Cowley, T.G. Perring, D.F. McMorrow, B. Roessli, Phys. Rev. B 57, 5281 (1998).

[2] Shan-Ho Tsai, A. Bunker, D.P. Landau, Phys. Rev. $B$ 61, 333 (2000).

[3] T. Hubermann, D.A. Tennant, R.A. Cowley, R. Coldea, C.D. Frost.

[4] R.A. Cowley, G. Shirane, R.J. Birgeneau, H.J. Guggenheim, Phys. Rev. B 15, 4192 (1977).

[5] T. Huberman, R. Coldea, R.A. Cowley, D.A. Tennant, R.L. Leheny, R.J. Christianson, C.D. Frost, Phys. Rev. B 72, 014413 (2005).

[6] N. Metropolis, A.W. Rosenbluth, M.N. Rosenbluth, A.H. Teller, E. Teller, J. Chem. Phys. 21, 1087 (1953).

[7] S. Tyc, B.I. Halperin, Phys. Rev. B 42, 2096 (1990).

[8] P. Kopietz, Phys. Rev. B 41, 9228 (1990).

[9] R. Freedman, G.F. Mazenko, Phys. Rev. B 13, 4967 (1976).

[10] A. Cuccoli, S.W. Lovesey, V. Tognetti, J. Phys., Condens. Matter 6, 7553 (1994).

[11] Y.S. Lee, M. Greven, B.O. Wells, G. Shirane, Euro Phys. B 5, 15 (1998).

[12] P. Hasenfratz, Euro Phys. 268, 231 (2000).

[13] R. Christianson, R. Leheny, R.J. Birgeneau, R.W. Erwin, Phys. Rev. B 63, 140401 (2001). 